\title{
Relational Interpretations of Neighborhood Operators and Rough Set Approximation Operators
}

\author{
Y.Y. Yao \\ Department of Computer Science, Lakehead University, Thunder Bay, Ontario, \\ Canada P7B 5E1, E-mail: yyao@flash.lakeheadu.ca
}

This paper presents a framework for the formulation, interpretation, and comparison of neighborhood systems and rough set approximations using the more familiar notion of binary relations. A special class of neighborhood systems, called 1-neighborhood systems, is introduced. Three extensions of Pawlak approximation operators are analyzed. Properties of neighborhood and approximation operators are studied, and their connections are examined.

Key words: Approximation operators, binary relations, neighborhood systems, rough sets, partitions, coverings.

\section{INTRODUCTION}

The theory of rough sets is motivated by practical needs in classification, concept formation, and data analysis with insufficient and incomplete information [12-15]. It provides a systematic approach for the study of indiscernibility of objects. Typically, indiscernibility is described using equivalence relations. When objects of a universe are represented by using a set of attributes, one may define the indiscernibility of objects based on their attribute values. If two objects are characterized by the same values on certain attributes, i.e., they have the same description, they are said to be indistinguishable or equivalent. All objects with the same description form an equivalence class. The family of equivalence classes defines a partition of the universe. In terms of equivalence classes, a subset of the universe may be approximated by two subsets. The lower approximation is the union of equivalence classes which are subsets of the given set, and the upper approximation is the union of equivalence classes which have a nonempty intersection with the given set. They can be formally described by a pair of unary set-theoretic operators [23]. By applying the argument in a wider context, one may generalize the notion of approximation 
operators by using non-equivalence relations [10,19,20,22,24], or a covering of the universe $[10,16,25]$. This leads to various approximation operators.

By adopting the notion of neighborhood systems from topological space and its generalization called Frechet (V)space [18], Lin [4,5,7,8] proposed a more general framework for the study of approximation. In neighborhood systems, each element of a universe is associated with a nonempty family of subsets of the universe. This family is called a neighborhood system of the element, and each member is called a neighborhood of the element. A subset of the universe can be approximated based on neighborhood systems of all elements in the universe. With respect to an equivalence relation, the equivalence class containing a given element may be interpreted as a neighborhood of that element $[10,20]$. For an arbitrary binary relation, the successor elements of a given element may be interpreted as its neighborhood [24]. The theory of rough sets built from binary relations may therefore be related to neighborhood systems.

The main objective of this paper is to build a common framework for the study of a special class of neighborhood systems and rough set approximations. Binary relations are used as a primitive notion to interpret various concepts involved. Within the proposed framework, main results of studies on rough set approximation operators are reviewed. Pawlak approximation operators are extended in three directions. One extension is consistent with the interpretation of necessity and possibility operators of modal logic. The resulting approximation operators can be expressed in terms of neighborhood operators. The other two methods use coverings of universe induced by neighborhood systems. The connections between binary relations, neighborhood operators, and approximation operators are examined.

\section{RELATION BASED NEIGHBORHOOD OPERATORS}

Let $U$ denote a finite and nonempty set called the universe. For each element $x$ of $U$, one associates it with a subset $n(x) \subseteq U$ called a neighborhood of $x$. A neighborhood of $x$ may or may not contain $x$. A neighborhood system $\mathrm{NS}(x)$ of $x$ is a nonempty family of neighborhoods of $x$. A neighborhood system of $U$, denoted by $\mathrm{NS}(U)$, is the collection of $\mathrm{NS}(x)$ for all $x$ in $U$. It determines a Frechet $(\mathrm{V})$ space, written $(U, \mathrm{NS}(U))$. There is no additional requirement on the neighborhood systems in a Frechet (V)space. A topological space is a Frechet $(\mathrm{V})$ space, but the converse is not true $[5,6]$.

In this study, we consider a special type of neighborhood systems in which each element has exactly one neighborhood. They are called 1-neighborhood systems. Such a neighborhood system can be described by a neighborhood 
operator $n: U \longrightarrow 2^{U}$, where $2^{U}$ denotes the power set of the universe. The operator $n$ assigns a unique neighborhood $n(x)$ to each element $x \in U$. For a finite universe $U$, we can extend a neighborhood operator $n$ from $U$ to $2^{U}$ as follows:

$$
N(X)=\bigcup_{x \in X} n(x)
$$

For the empty set $\emptyset$, we define $N(\emptyset)=\emptyset$. The mapping $N: 2^{U} \longrightarrow 2^{U}$ associates each subset of $U$ with a subset of $U$. It may be considered as an unary set-theoretic operator. By definition, we have $N(\{x\})=n(x)$. Operator $N$ may therefore be interpreted as an additive extension of $n$.

In order to study the structures of 1-neighborhood systems, we consider the following properties of a neighborhood operator:

serial : for all $x \in U$, there exists a $y \in U$ such that $y \in n(x)$, for all $x \in U, n(x) \neq \emptyset$,

inverse serial : for all $x \in U$, there exists a $y \in U$ such that $x \in n(y)$,

$$
\bigcup_{x \in U} n(x)=U
$$

reflexive : for all $x \in U, x \in n(x)$,

symmetric : for all $x, y \in U, x \in n(y) \Longrightarrow y \in n(x)$,

transitive : for all $x, y, z \in U,[y \in n(x), z \in n(y)] \Longrightarrow z \in n(x)$,

Euclidean : for all $x, y, z \in U,[y \in n(x), z \in n(x)] \Longrightarrow z \in n(y)$.

A reflexive neighborhood operator is both serial and inverse serial. The family of neighborhoods $\{n(x) \mid x \in U\}$ of an inverse serial neighborhood operator forms a covering of the universe. By combining these properties, we can characterize more classes of neighborhood systems [8]. A neighborhood system is called a B-neighborhood system if the neighborhood operator is reflexive and symmetric. It is called a Pawlak-neighborhood system if the neighborhood operator is reflexive, symmetric, and transitive [5,8]. A Pawlak-neighborhood system can be equivalently characterized by the properties of reflexivity and Euclidean. The family of neighborhoods of a Pawlak neighborhood operator forms a partition of the universe.

The class of 1-neighborhood systems can be interpreted using the more familiar notion of binary relations. A binary relation $R$ over a universe $U$ is a subset of the Cartesian product $U \times U$. For two elements $x, y \in U$, if $x R y$, we say that $y$ is $R$-related to $x, x$ is a predecessor of $y$, and $y$ is a successor of $x$. Given a binary relation, we define the successor neighborhood of $x$ as follows:

$$
R_{s}(x)=\{y \mid x R y\} .
$$


It can be viewed as a successor neighborhood operator from $U$ to $2^{U}$. Properties of a binary relation $R$ can be stated using the successor neighborhood operator:

serial : for all $x \in U$, there exists a $y \in U$ such that $x R y$, for all $x \in U, R_{s}(x) \neq \emptyset$,

inverse serial : for all $x \in U$, there exists a $y \in U$ such that $y R x$,

$$
\bigcup_{x \in U} R_{s}(x)=U
$$

reflexive : for all $x \in U, x R x$,

for all $x \in U, x \in R_{s}(x)$,

symmetric : for all $x, y \in U, x R y \Longrightarrow y R x$,

for all $x, y \in U, x \in R_{s}(y) \Longrightarrow y \in R_{s}(x)$,

transitive : for all $x, y, z \in U,[x R y, y R z] \Longrightarrow x R z$,

for all $x, y, z \in U,\left[y \in R_{s}(x), z \in R_{s}(y)\right] \Longrightarrow z \in R_{s}(x)$,

for all $x, y \in U, y \in R_{s}(x) \Longrightarrow R_{s}(y) \subseteq R_{s}(x)$,

Euclidean : for all $x, y, z \in U,[x R y, x R z] \Longrightarrow y R z$,

for all $x, y, z \in U,\left[y \in R_{s}(x), z \in R_{s}(x)\right] \Longrightarrow z \in R_{s}(y)$,

for all $x, y \in U, y \in R_{s}(x) \Longrightarrow R_{s}(x) \subseteq R_{s}(y)$.

They correspond to the properties of neighborhood operators. For this reason, we have in fact used the same naming system for both binary relations and neighborhood operators.

Let $R$ and $Q$ be two binary relations. We define operations on binary relations through set-theoretic operations:

$$
\begin{aligned}
\sim R & =\{(x, y) \mid \text { not } x R y\}, \\
R \cap Q & =\{(x, y) \mid x R y \text { and } x Q y\}, \\
R \cup Q & =\{(x, y) \mid x R y \text { or } x Q y\} .
\end{aligned}
$$

They are referred to as the complement, intersection, and union of binary relations, respectively. For two relations $R$ and $Q$, the successor neighborhoods defined by $\sim R, R \cap Q$, and $R \cup Q$ are given by:

$$
\begin{aligned}
(\sim R)_{s}(x) & =\sim R_{s}(x), \\
(R \cap Q)_{s}(x) & =R_{s}(x) \cap Q_{s}(x), \\
(R \cup Q)_{s}(x) & =R_{s}(x) \cup Q_{s}(x) .
\end{aligned}
$$

They follow from the definitions of operations on binary relations and successor neighborhoods. The set inclusion defines an order on binary relations on $U$. A relation $R$ is said to be finer than another relation $Q$, i.e., $Q$ is coarser than $R$, if $R \subseteq Q$. The successor neighborhoods of a finer relation are smaller than that of a coarser relation, namely: 


$$
R \subseteq Q \Longleftrightarrow \text { for all } x \in U, R_{s}(x) \subseteq Q_{s}(x) .
$$

Relation $R \cap Q$ is finer than both $R$ and $Q$, they are finer than $R \cup Q$.

With a binary relation, we can define additional types of neighborhoods of $x$ :

$$
\begin{aligned}
R_{p}(x) & =\{y \mid y R x\}, \\
R_{p \wedge s}(x) & =\{y \mid x R y \text { and } y R x\}=R_{p}(x) \cap R_{s}(x), \\
R_{p \vee s}(x) & =\{y \mid x R y \text { or } y R x\}=R_{p}(x) \cup R_{s}(x) .
\end{aligned}
$$

They are called the predecessor, predecessor-and-successor, and predecessoror-successor neighborhood operators, respectively. Relationships between these neighborhood system can be expressed as:

$$
\begin{aligned}
& R_{p \wedge s}(x) \subseteq R_{p}(x) \subseteq R_{p \vee s}(x), \\
& R_{p \wedge s}(x) \subseteq R_{s}(x) \subseteq R_{p \vee s}(x) .
\end{aligned}
$$

A binary relation and neighborhood operators $R_{p}$ and $R_{s}$ uniquely determine each other, namely,

$$
x R y \Longleftrightarrow x \in R_{p}(y) \Longleftrightarrow y \in R_{s}(x) .
$$

However, it is impossible to define others from operators $R_{p \wedge s}$ and $R_{p \vee s}$. For a symmetric relation, all neighborhood operators $R_{p}, R_{s}, R_{p \wedge s}$, and $R_{p \vee s}$ reduce to the same one. Relationships between properties of a binary relation and the neighborhood operators are summarized in Table 1. The entry "i. serial" stands for inverse serial, and the entry "serial \& i." stands for serial and inverse serial. For reflexive and symmetric relations, all four neighborhood operators have the same property of the corresponding binary relations.

Binary relation based neighborhoods have been studied by many authors. Orlowska [9-11,20] viewed $R_{p \vee s}(x)$ as a neighborhood of $x$. Yao and Lin [24] regarded $R_{s}(x)$ as a neighborhood of $x$. In general, one may define additional neighborhood operators, such as $R_{\neg s}, R_{\neg p}, R_{\neg p \vee s}$, etc., which are selfexplanatory. The family of all possible neighborhood operators induced by a binary relation forms an atomic Boolean algebra, in which the set of atoms consists of $R_{\neg p \wedge \neg s}, R_{\neg p \wedge s}, R_{p \wedge \neg s}$, and $R_{p \wedge s}$. There are in total 16 different neighborhood operators. Each of them represents a different interpretation of the notion of neighborhoods of an element of $U$. Their additive extensions provide neighborhoods of subsets of $U$.

For a binary relation $R$, its inverse $R^{-1}$ is a binary relation defined by [2]:

$$
y R^{-1} x \Longleftrightarrow x R y
$$


Table 1

Properties of a binary relation and induced neighborhood operators

\begin{tabular}{|l||llll|}
\hline$R$ & $R_{s}$ & $R_{p}$ & $R_{p \wedge s}$ & $R_{p \vee s}$ \\
\hline any & & & symmetric & symmetric \\
serial & serial & i. serial & & serial \& i. \\
i. serial & i. serial & serial & & serial \& i. \\
reflexive & reflexive & reflexive & reflexive & reflexive \\
symmetric & symmetric & symmetric & symmetric & symmetric \\
transitive & transitive & transitive & transitive & \\
Euclidean & Euclidean & & Euclidean & \\
\hline
\end{tabular}

$R$ is reflexive and transitive if and only if $R^{-1}$ is reflexive and transitive, respectively. $R$ is a symmetric if and only if $R=R^{-1}$. In this case, we have $R=R^{-1}=R \cap R^{-1}=R \cup R^{-1}$. Independent of the properties of $R, R \cap R^{-1}$ and $R \cup R^{-1}$ are symmetric relations. For a relation $R$ and its inverse $R^{-1}$, the application of operators $\sim, \cap$, and $\cup$ produces 16 different relations, such as $\sim R, \sim R^{-1}$, and $R \cup \sim R^{-1}$. The predecessor neighborhood defined by $R$ is the successor neighborhood defined by $R^{-1}$, namely,

$$
R_{p}(x)=\{y \mid y R x\}=\left\{y \mid x R^{-1} y\right\}=R_{s}^{-1}(x) .
$$

Combining this result with equations (4) and (6), one can establish an oneto-one correspondence between different neighborhood operators induced by $R$ with the successor neighborhoods induced by relations derived from $R$ and $R^{-1}$. For example, $R_{p \wedge s}$ and $R_{p \vee s}$ can be expressed in terms of successor operator as follows:

$$
\begin{aligned}
& R_{p \wedge s}(x)=\left(R \cap R^{-1}\right)_{s}(x), \\
& R_{p \vee s}(x)=\left(R \cup R^{-1}\right)_{s}(x) .
\end{aligned}
$$

We therefore have an alternative method for the formulation of neighborhood operators with respect to a binary relation.

Neighborhood operators can be extended to subsets of universe by additive extension. For example, for the successor neighborhood operator we have: 


$$
\begin{aligned}
R_{s}(X) & =\bigcup_{x \in X} R_{s}(x) \\
& =\{y \mid \text { there exists a } x \in X \text { such that } x R y\}
\end{aligned}
$$

where the same symbol is used to denote both neighborhood operators. The same relationships between neighborhood operators can be expressed using subsets of $U$. For instance, for $X \subseteq U$ we have:

$$
\begin{aligned}
R_{p}(X) & =R_{s}^{-1}(X), \\
R_{p \wedge s}(X) & =\left(R \cap R^{-1}\right)_{s}(X), \\
R_{p \vee s}(X) & =\left(R \cup R^{-1}\right)_{s}(X) .
\end{aligned}
$$

They are counterparts of equations (10) and (11).

\section{ROUGH SET APPROXIMATION OPERATORS}

In this section, we review and generalize Pawlak approximation operators based on the notion of 1-neighborhood systems.

\subsection{PAWLAK ROUGH SETS}

Let $R \subseteq U \times U$ be an equivalence relation on $U$, i.e., $R$ is reflexive, symmetric, and transitive. The pair $a p r=(U, R)$ is called a Pawlak approximation space. The equivalence relation $R$ partitions the universe $U$ into disjoint subsets called equivalence classes. Elements in the same equivalence class are said to be indistinguishable. Equivalent classes of $R$ are called elementary sets. A union of elementary sets is called a definable (composed) set [12,13]. The empty set is considered to be a definable set [23]. The family of all definable sets is denoted by $\operatorname{Def}(a p r)$. A Pawlak approximation space defines uniquely a topological space $(U, \operatorname{Def}(a p r))$, in which $\operatorname{Def}(a p r)$ is the family of all open and closed sets [12].

Given a subset $X \subseteq U$, one can approximate $X$ by a pair of subsets of $U$. The lower approximation $\operatorname{apr}(X)$ is the greatest definable set contained in $X$, and the upper approximation $\overline{a p r}(X)$ is the least definable set containing $X$. They correspond to the interior and closure of $X$ in the topological space $(U, \operatorname{Def}(a p r))$, and are dual to each other:
(a) $\operatorname{apr}(X)=\sim \overline{a p r}(\sim X)$,
(b) $\overline{\overline{a p r}}(X)=\sim \operatorname{apr}(\sim X)$. 
One may interpret apr, $\overline{a p r}: 2^{U} \longrightarrow 2^{U}$ as a pair of dual unary set-theoretic operators $[6,22]$. The system $\left(2^{U}, \cap, \cup, \sim, \underline{a p r}, \overline{a p r}\right)$ is called a Pawlak rough set algebra defined by the equivalence relation $R$. It may be viewed as an extension of classical set algebra $\left(2^{U}, \cap, \cup, \sim\right)$.

In the development of rough set theory, two additional and distinct interpretations of approximation operators have been proposed. One is focused on the elements of $U$, and the other on the equivalence classes of $R$. An element $x \in U$ belongs to the lower approximation of $X$ if all its equivalent elements belong to $X$. It belongs to the upper approximation of $X$ if at least one of its equivalent elements belongs to $X$. That is,

$$
\text { (i) } \begin{aligned}
\underline{\operatorname{apr}}(X) & =\left\{x \in U \mid[x]_{R} \subseteq X\right\} \\
& =\{x \in U \mid \text { for all } y \in U, x R y \text { implies } y \in X\} \\
& =\left\{x \in U \mid \forall y\left[y \in[x]_{R} \Longrightarrow y \in X\right]\right\}, \\
\overline{\operatorname{apr}}(X) & =\left\{x \in U \mid[x]_{R} \cap X \neq \emptyset\right\} \\
& =\{x \in U \mid \text { there exists a } y \in U \text { such that } x R y \text { and } y \in X\} \\
& =\left\{x \in U \mid \exists y\left[y \in[x]_{R}, y \in X\right]\right\},
\end{aligned}
$$

where

$$
[x]_{R}=\{y \mid x R y\}
$$

is the equivalence class containing $x$. This interpretation of approximation operators is related to interpretation of the necessity and possibility operators in modal logic $[23,24]$. Alternatively, in terms of equivalence classes of $R$, the pair of lower and upper approximation operators can be defined by:

$$
\begin{aligned}
& \underline{\operatorname{apr}}(X)=\bigcup\left\{[x]_{R} \mid x \in U,[x]_{R} \subseteq X\right\}, \\
& \overline{\operatorname{apr}}(X)=\bigcup\left\{[x]_{R} \mid x \in U,[x]_{R} \cap X \neq \emptyset\right\} .
\end{aligned}
$$

The lower approximation of $X$ is the union of equivalence classes that are subsets of $X$, and the upper approximation is the union of equivalence classes that have a nonempty intersection with $X$.

If non-equivalence binary relations are used, the two interpretations (i) and (ii) provide different generalizations of approximation operators [9-11,16,17,2225]. They are discussed in the following subsections. 


\subsection{APPROXIMATION AND NEIGHBORHOOD OPERATORS}

In generalizing Pawlak approximation operators, we may therefore use different neighborhood operators to define distinct approximation operators. For an equivalence relation $R$, the equivalence class $[x]_{R}$ may be considered as a neighborhood of $x$. Let $n$ denote an arbitrary neighborhood operator and $n(x)$ the corresponding neighborhood of $x$. By substituting $[x]_{R}$ with $n(x)$ in definition (i), we define a pair of approximation operators $[4,6,23]$ :

(I)

$$
\begin{aligned}
\underline{\operatorname{apr}}_{n}(X) & =\{x \in U \mid n(x) \subseteq X\} \\
& =\{x \in U \mid \text { for all } y \in U, y \in n(x) \text { implies } y \in X]\} \\
& =\{x \in U \mid \forall y[y \in n(x) \Longrightarrow y \in X]\}, \\
\overline{\operatorname{apr}}_{n}(X) & =\{x \in U \mid n(x) \cap X \neq \emptyset\} \\
& =\{x \in U \mid \text { there exists a } y \in U \text { such that } y \in n(x) \text { and } y \in X\} \\
& =\{x \in U \mid \exists y[y \in n(x), y \in X]\} .
\end{aligned}
$$

The subscript $n$ indicates that the approximation operators are defined based on a particular neighborhood operator $n$. They can be viewed as a generalization of (i). The system $\left(2^{U}, \cap, \cup, \sim, \underline{a p r}_{n}, \overline{a p r}_{n}\right)$ is called a rough set algebra.

Theorem 1 For an arbitrary neighborhood operator, the pair of approximation operators satisfies the properties:

$$
\begin{aligned}
& \underline{\operatorname{apr}}_{n}(X)=\sim\left(\overline{\operatorname{apr}}_{n}(\sim X)\right), \\
& \overline{a p r}_{n}(X)=\sim\left(\underline{a p r}_{n}(\sim X)\right) ; \\
& \frac{a p r}{n}(U)=U, \\
& \overline{\operatorname{apr}}_{n}(\emptyset)=\emptyset ; \\
& \overline{a p r}_{n}(X \cap Y)=\overline{a p r}_{n}(X) \cap \overline{a p r}_{n}(Y), \\
& \overline{a p r}_{n}(X \cup Y)=\overline{a p r}_{n}(X) \cup \overline{a p r}_{n}(Y) .
\end{aligned}
$$

PROOF. It can be easily checked by definition that (L1) and (U1) hold. Approximation operators are defined by using the universal and existential quantifiers. The other two properties trivially follow from the following laws of predicate logic:

(a) $\quad \forall x[P(x)] \Longleftrightarrow \neg(\exists x[\neg P(x)])$,

$$
\exists x[P(x)] \Longleftrightarrow \neg(\forall x[\neg P(x)]),
$$

(b) $\quad \forall x[P(x) \wedge Q(x)] \Longleftrightarrow \forall x[P(x)] \wedge \forall x[Q(x)]$, $\exists x[P(x) \vee Q(x)] \Longleftrightarrow \exists x[P(x)] \vee \exists x[Q(x)]$. 
where the variable $x$ is over $U$, and $P$ and $Q$ are predicate symbols.

The interpretation of rough set approximation operators using predicate logic was recently studied by Wong and Yao [21].

Properties (L0) and (U0) show that approximation operators $\underline{\operatorname{apr}}_{n}$ and $\overline{a p r}_{n}$ are dual to each other. Properties with the same number may be considered as dual properties. The first three properties are independent. They also imply many other useful properties of approximation operators, for example,

$$
\begin{array}{ll}
\text { (L3) } & \underline{\operatorname{apr}}_{n}(X \cup Y) \supseteq \underline{a p r}_{n}(X) \cup \underline{\underline{a p r}}_{n}(Y), \\
\text { (U3) } & \overline{a p r}_{n}(X \cap Y) \subseteq \overline{a p r}_{n}(X) \cap \overline{\overline{a p r}}_{n}(Y) ; \\
\text { (L4) } & X \subseteq Y \Longrightarrow \underline{a p r}_{n}(X) \subseteq \underline{a p r}_{n}(Y), \\
\text { (U4) } & X \subseteq Y \Longrightarrow \overline{\operatorname{apr}}_{n}(X) \subseteq \overline{\operatorname{apr}}_{n}(Y) ; \\
\text { (L5) } & \underline{a p r}_{n}(X)=\bigcap_{x \notin X} \underline{a p r}_{n}(\sim\{x\}), \\
\text { (U5) } & \overline{a p r}_{n}(X)=\bigcup_{x \in X} \overline{a p r}_{n}(\{x\}) .
\end{array}
$$

Properties (L4) and (U4) state that approximation operators are monotonic with respect to set inclusion. Properties (L5) and (U5) show the relationships between the approximations of the set $X$ and the approximations of a family of subsets of $U$ constructed from the singleton subsets of $X$.

Additional properties of approximation operators are determined by the properties of neighborhood operators. The main results are summarized in the following theorem.

Theorem 2 Suppose $n: U \longrightarrow 2^{U}$ is a neighborhood operators. With respect to serial, inverse serial, reflexive, symmetric, transitive, and Euclidean neighborhood operators, the approximation operators have the following corresponding properties:

(U6) $\quad \overline{a p r}_{n}(U)=U$,

(LU6) $\quad \underline{a p r}_{n}(X) \subseteq \overline{a p r}_{n}(X)$;

(L7) for all $x \in U, \underline{a p r}_{n}(\sim\{x\}) \neq U$;

(U7) for all $x \in U, \overline{a p r}_{n}(\{x\}) \neq \emptyset$;

(L8) $\quad \underline{a p r}_{n}(X) \subseteq X$,

(U8) $\quad X \subseteq \overline{a p r}_{n}(X)$;

(L9) $X \subseteq \underline{a p r}_{n}\left(\overline{a p r}_{n}(X)\right)$,

(U9) $\quad \overline{a p r}_{n}\left(\underline{a p r}_{n}(X)\right) \subseteq X$; 


$$
\begin{aligned}
& \underline{a p r}_{n}(X) \subseteq \underline{a p r}_{n}\left(\underline{a p r}_{n}(X)\right), \\
& \overline{a p r}_{n}\left(\overline{a p r}_{n}(X)\right) \subseteq \overline{a p r}_{n}(X) ; \\
& \overline{a p r}_{n}(X) \subseteq \underline{a p r}_{n}\left(\overline{a p r}_{n}(X)\right), \\
& \left.\overline{a p r}_{n}\left(\underline{a p r}_{n}(X)\right) \subseteq \underline{a p r}_{n}(X)\right) .
\end{aligned}
$$

PROOF. By the duality of approximation operators, we only need to prove one of the dual properties.

Serial neighborhood operator: For any $x \in U$, we have $n(x) \neq \emptyset$. It immediately follows that (L6) holds. Now suppose $x \in \underline{a p r}_{n}(X)$. We have $n(x) \subseteq X$ and $n(x) \neq \emptyset$. They imply $n(x) \cap X \neq \emptyset$, namely, $x \in \overline{a p r}_{n}(X)$. Thus, property (LU6) holds. In fact, one can easily show that (L6), (U6), and (LU6) are equivalent, provided that (L0), (U0), (L2), and (U2) hold.

Inverse serial neighborhood operator: For any $x \in U$, there must exist a $y \in U$ such that $x \in n(y)$. Hence, $n(y) \nsubseteq \sim\{x\}$. By definition, $y$ must not belong to $\underline{\operatorname{apr}}_{n}(\sim\{x\})$, which implies that (L7) holds.

Reflexive neighborhood operator: For any $x \in U, x \in n(x)$. Suppose $x \in$ $\underline{\operatorname{apr}}_{n}(X)$, which is equivalent to $n(x) \subseteq X$. Combining $x \in n(x)$ and $n(x) \subseteq X$, we have $x \in X$. Thus, (L8) holds.

Symmetric neighborhood operator: Suppose $x \in X$. By the symmetry of $n$, for all $y \in n(x)$ we have $x \in n(y)$, i.e., $x \in n(y) \cap X$. This implies that for all $y \in n(x), y \in \overline{a p r}_{n}(X)$. Hence, $n(x) \subseteq \overline{a p r}_{n}(X)$, which means that $x \in \underset{\underline{a p r}_{n}}{ }\left(\overline{\operatorname{apr}}_{n}(X)\right)$. Therefore, (L9) holds.

Transitive neighborhood operator: Suppose $x \in \underline{a p r}_{n}(X)$, i.e., $n(x) \subseteq X$. By transitivity, for all $y \in n(x), n(y) \subseteq n(x) \subseteq X$. This is equivalent to say that for all $y \in n(x), y \in \underline{\operatorname{apr}}_{n}(X)$. One can therefore conclude that $n(x) \subseteq \underline{\operatorname{apr}}_{n}(X)$ and in turn $\left.x \in \underline{a p r} \underline{n}_{n} \underline{a p r}_{n}(X)\right)$. That is, (L10) holds.

Euclidean neighborhood operator: Suppose $x \in \overline{a p r}_{n}(X)$, i.e., $n(x) \cap X \neq \emptyset$. By the Euclidean property of $n$, for all $y \in n(x), n(x) \subseteq n(y)$. Combining this result with $n(x) \cap X \neq \emptyset$, we can conclude that for all $y \in n(x), y \in \overline{a p r}_{n}(X)$. This is equivalent to say $n(x) \subseteq \overline{a p r}_{n}(X)$, which implies $x \in \underline{a p r}_{n}\left(\overline{a p r}_{n}(X)\right)$. Therefore, (L11) holds.

Approximation operators defined by (I) are consistent with the notion of necessity and possibility operators in modal logic $[11,23,24]$. With respect to axioms in modal logic [1], if necessity operator $\square$ is replaced by $\underline{a p r}_{n}$, possibility operator $\diamond$ by $\overline{a p r}_{n}$, negation $\neg$ by set complement $\sim$, conjunction $\wedge$ by set intersection $\cap$, disjunction $\vee$ by set union $\cup$, and implication $\rightarrow$ by 
set inclusion $\subseteq$, one can obtain the axioms of approximation operators. Most of the properties of approximation operators discussed so far correspond to axioms in modal logic.

With respect to a binary relation, we may define distinct approximation operators by using different neighborhood operators. The choice of a particular rough set algebra depends on the application. For neighborhood operators $R_{p}(x), R_{s}(x), R_{p \wedge s}(x)$, and $R_{p \vee s}(x)$, four commonly used pairs of approximation operators are defined by:

$$
\begin{aligned}
& \frac{a p r}{R_{p}}(X)=\left\{x \in U \mid R_{p}(x) \subseteq X\right\}, \\
& \overline{a p r}_{R_{p}}(X)=\left\{x \in U \mid R_{p}(x) \cap X \neq \emptyset\right\}, \\
& \frac{a p r}{R_{s}}(X)=\left\{x \in U \mid R_{s}(x) \subseteq X\right\}, \\
& \overline{a p r}_{R_{s}}(X)=\left\{x \in U \mid R_{s}(x) \cap X \neq \emptyset\right\}, \\
& \overline{a p r}_{R_{p \wedge s}}(X)=\left\{x \in U \mid R_{p \wedge s}(x) \subseteq X\right\}, \\
& \overline{a p r}_{R_{p \wedge s}}(X)=\left\{x \in U \mid R_{p \wedge s}(x) \cap X \neq \emptyset\right\}, \\
& \overline{a p r}_{R_{p \vee s}}(X)=\left\{x \in U \mid R_{p \vee s}(x) \subseteq X\right\}, \\
& \overline{a p r}_{R_{p \vee s}}(X)=\left\{x \in U \mid R_{p \vee s}(x) \cap X \neq \emptyset\right\} .
\end{aligned}
$$

For Pawlak approximation operators, an equivalence relation $R$ is used. In this case, four neighborhood operators become the same, i.e., $R_{p}(x)=R_{s}(x)=$ $R_{p \wedge s}(x)=R_{p \vee s}(x)=[x]_{R}$. All definitions (I1)-(I4) are equivalent. The condition of an equivalence relation is sufficient but not necessary. A necessary and sufficient condition is given below.

Theorem 3 Two pairs of lower and upper approximation operators from (I1)(I4) are equivalent if and only if the binary relation $R$ is symmetric.

PROOF. $(\Longleftarrow)$ If $R$ is a symmetric relation, we have for $R_{p}(x)=R_{s}(x)=$ $R_{p \wedge s}(x)=R_{p \vee s}(x)$. By definition, (I1)-(I4) are equivalent.

$(\Longrightarrow)$ Consider the upper approximation of singleton subsets of $U$. By definition, for approximation operator $\overline{a p r}_{R_{p}}$, we have:

$$
\begin{aligned}
\overline{\operatorname{apr}}_{R_{p}}(\{x\}) & =\left\{y \mid R_{p}(y) \cap\{x\} \neq \emptyset\right\} \\
& =\left\{y \mid x \in R_{p}(y)\right\} \\
& =\{y \mid x R y\} \\
& =\left\{y \mid y \in R_{s}(x)\right\} \\
& =R_{s}(x) .
\end{aligned}
$$

Similarly, 


$$
\begin{aligned}
& \overline{a p r}_{R_{s}}(\{x\})=R_{p}(x), \\
& \overline{a p r}_{R_{p \wedge s}}(\{x\})=R_{p \wedge s}(x), \\
& \overline{a p r}_{R_{p \vee s}}(\{x\})=R_{p \vee s}(x) .
\end{aligned}
$$

Suppose (I1) and (I2) are equivalent, we must have: for all $x \in U$,

$$
R_{s}(x)=\overline{a p r}_{R_{p}}(\{x\})=\overline{a p r}_{R_{s}}(\{x\})=R_{p}(x) .
$$

Therefore, we can conclude that $R$ is symmetric. Following the same argument, we can show that the equivalence of any pair from (I1)-(I4) implies that $R$ is a symmetric relation.

If a binary relation is not symmetric, one may obtain distinct approximation operators. By definition, each neighborhood operator defines a pair of dual approximation operators. Orlowska [11] used definition (I1) in the investigation of dynamic information systems involving temporal information. Definition (I4) was used by Orlowska [9-11] and Wasilewska [20]. The operators given by (I2) is a commonly used definition, which has been investigated by many authors either explicitly through a binary relation or implicitly through a covering of the universe $[11,16,17,22,24]$. A number of proposals have been made for using approximation operators that are not dual to each other. Slowinski and Vanderpooten [19] used $\underline{a p r}_{R_{p}}$ and $\overline{a p r}_{R_{s}}$ as a pair of approximation operators in the study of rough approximations using binary relations that are only reflexive. Wybraniec-Skardowska [22] used $\underline{a p r} \underline{R}_{s}$ and $\overline{a p r}_{R_{p}}$ as a pair of approximations determined by a binary relation. In this study, we only consider dual approximation operators.

According to property (U2) and equations (15) and (16), upper approximation operators can be interpreted using neighborhood operators:

$$
\begin{aligned}
& \overline{a p r}_{R_{p}}(X)=R_{s}(X), \\
& \overline{a p r}_{R_{s}}(X)=R_{p}(X), \\
& \overline{a p r}_{R_{p \wedge s}}(X)=R_{p \wedge s}(X), \\
& \overline{a p r}_{R_{p \vee s}}(X)=R_{p \vee s}(X) .
\end{aligned}
$$

Clearly, rough set approximation operators are in fact neighborhood operators. The upper approximation of $X$ defined by the predecessor neighborhood operator is the successor neighborhood of $X$, while the upper approximation of $X$ defined by the successor neighborhood operator is the predecessor neighborhood of $X$. In contrast, operators $R_{p \wedge s}$ and $R_{p \vee s}$ produce upper approximations that are the same as the corresponding neighborhoods. For these operators, we can establish the following relationships: 


$$
\begin{aligned}
& \underline{\operatorname{apr}}_{R_{p \wedge s}}(X)=\left\{x \in U \mid \overline{a p r}_{R_{p \wedge s}}(\{x\}) \subseteq X\right\}, \\
& \underline{a p r}_{R_{p \vee s}}(X)=\left\{x \in U \mid \overline{a p r}_{R_{p \vee s}}(\{x\}) \subseteq X\right\} .
\end{aligned}
$$

This connection between lower and upper approximation operators has been investigated by some authors [17,22]. Wybraniec-Skardowska [22] defined a lower approximation operator in terms of upper approximations of singleton subsets of $U$ using equation (19). One can verify that there does not exist such a relationship between $\frac{a p r}{R_{p}}$ and $\overline{a p r}_{R_{p}}$, and between $\underline{a p r}_{R_{s}}$ and $\overline{a p r}_{R_{s}}$. This implies that in general one may not use equation (19) to define dual approximation operators.

\subsection{APPROXIMATION OPERATORS AND NEIGHBORHOODS}

In a Pawlak approximation space, the family of equivalence classes forms a partition of universe. By using coverings instead of partitions, many studies generalize Pawlak approximation operators $[16,22,25]$. Such a generalization can be easily interpreted in the framework of neighborhood systems.

Using 1-neighborhood systems, we may define a pair of approximation operators by replacing the equivalence class $[x]_{R}$ with the neighborhood $n(x)$ in definition (ii). However, there exists a problem with such a straightforward extension. The lower and upper approximation operators are not necessarily dual operators. To resolve this problem, one may extend definition (ii) in two ways. Either the lower or the upper approximation operator may be extended, and the other one is defined by duality. The results are two pairs of dual approximation operators:

$$
\begin{aligned}
\left(\mathrm{II}^{\prime}\right) \quad \underline{a p r}_{n}^{\prime}(X) & =\bigcup\{n(x) \mid x \in U, n(x) \subseteq X\} \\
& =\{x \in U \mid \exists y[x \in n(y), n(y) \subseteq X]\}, \\
\overline{a p r}_{n}^{\prime}(X) & =\sim{ }^{a p r^{\prime}}(\sim X) \\
& =\sim\{x \in U \mid \exists y[x \in n(y), n(y) \subseteq \sim X]\}, \\
& =\sim\{x \in U \mid \exists y[x \in n(y), n(y) \cap X=\emptyset]\}, \\
& =\{x \in U \mid \forall y[x \in n(y) \Longrightarrow n(y) \cap X \neq \emptyset]\}, \\
\left(\mathrm{II}^{\prime \prime}\right) \quad \underline{a p r}_{n}^{\prime \prime}(X) & =\sim \overline{a p r}_{n}^{\prime \prime}(\sim X) \\
& =\sim\{x \in U \mid \exists y[x \in n(y), n(y) \cap \sim X \neq \emptyset]\} \\
& =\{x \in U \mid \forall y[x \in n(y) \Longrightarrow n(y) \cap \sim X=\emptyset]\} \\
& =\{x \in U \mid \forall y[x \in n(y) \Longrightarrow n(y) \subseteq X]\}, \\
\overline{a p r}_{n}^{\prime \prime}(X) & =\bigcup\{n(x) \mid x \in U, n(x) \cap X \neq \emptyset\} \\
& =\{x \in U \mid \exists y[x \in n(y), n(y) \cap X \neq \emptyset]\} .
\end{aligned}
$$


They were suggested and studied, together with several pairs of dual approximation operators, by Pomykala $[16,17]$ using the notion of coverings. Properties of these approximation operators are summarized below.

Theorem 4 Suppose $n: U \longrightarrow 2^{U}$ is an arbitrary neighborhood operator. Approximation operators $\underline{a p r}_{n}^{\prime}$ and $\overline{a p r}_{n}^{\prime}$ satisfy (L0), (U0), (L8), (U8), (L10), (U10), and the following weaker versions of (L2) and (U2):

$$
\begin{aligned}
& {\underline{a p r}_{n}^{\prime}}_{n}(X \cap Y) \subseteq \underline{a p r}_{n}^{\prime}(X) \cap \underline{a p r}_{n}^{\prime}(Y), \\
& \overline{a p r}_{n}^{\prime}(X \cup Y) \supseteq \overline{a p r}_{n}^{\prime}(X) \cup \overline{a p r}_{n}^{\prime}(Y) .
\end{aligned}
$$

Approximation operators $\underline{a p r}_{n}^{\prime \prime}$ and $\overline{a p r}_{n}^{\prime \prime}$ satisfy (L0)-(L2), (U0)-(U2), (L9),

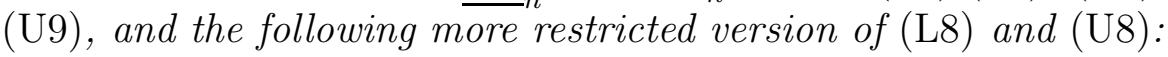

$$
\begin{aligned}
& \underline{a p r}_{n}^{\prime \prime}\left(\underline{a p r}_{n}^{\prime \prime}(X)\right) \subseteq \underline{a p r}_{n}^{\prime \prime}(X), \\
& \overline{a p r}_{n}^{\prime \prime}(X) \subseteq \overline{a p r}_{n}^{\prime \prime}\left(\overline{a p r}_{n}^{\prime \prime}(X)\right) .
\end{aligned}
$$

PROOF. All these properties can be easily checked by definitions (II') and $\left(\mathrm{II}^{\prime \prime}\right)$. As examples, we prove that $\underline{a p r}_{n}^{\prime}$ satisfies (L10), and $\underline{a p r_{n}^{\prime \prime}}$ and $\overline{a p r}{ }_{n}^{\prime \prime}$ satisfy (L9).

Assume $x \in \operatorname{apr}_{n}^{\prime}(X)$. By definition, there must exist a $y \in U$ such that $x \in n(y)$ and $\overline{n(y)} \subseteq X$. Moreover, for all $z \in n(y)$ we have $z \in \underline{a p r}_{n}^{\prime}(X)$, which implies $n(y) \subseteq \operatorname{apr}_{n}^{\prime}(X)$. From $x \in n(y)$, we immediately have $x \in$ $\underline{a p r}_{n}^{\prime}\left(\underline{a p r}_{n}^{\prime}(X)\right)$. Therefore, $\underline{a p r}_{n}^{\prime}(X) \subseteq \underline{a p r}_{n}^{\prime}\left(\underline{a p r}_{n}^{\prime}(X)\right)$, namely, (L10) holds.

For a subset $X \subseteq U$, assume $x \in X$. By definition, we have $\overline{a p r}_{n}^{\prime \prime}(X)=$ $\bigcup\{n(z) \mid z \in U, n(z) \cap X \neq \emptyset\}$. From assumption $x \in X$, for any $y$ if $x \in n(y)$ then $x \in n(y) \cap X \neq \emptyset$. This implies $n(y) \subseteq \overline{a p r}_{n}^{\prime \prime}(X)$. By definition, we have $x \in \underline{\operatorname{apr}}_{n}^{\prime \prime}\left(\overline{\operatorname{apr}}_{n}^{\prime \prime}(X)\right)$, namely (L9) holds.

Theorem 5 Suppose $n: U \longrightarrow 2^{U}$ is an inverse serial neighborhood operator. Approximation operators apr' and $\overline{\text { apr }}_{n}^{\prime}$ satisfy (L1) and (U1). Approximation

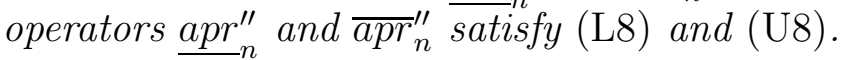

PROOF. For an inverse serial neighborhood operator $n$, the family $\{n(x) \mid$ $x \in U\}$ is a covering of the universe. It easily follows that $\operatorname{apr}_{n}^{\prime}$ satisfies (L1). For a subset $X \subseteq U$, assume $x \in X$. Since $n$ is an inverse serial neighborhood operator, there must exist a $y \in U$ such that $x \in n(y)$. We have $x \in X \cap n(y) \neq$ $\emptyset$. By definition, $x \in \overline{a p r}_{n}^{\prime \prime}(X)$. Thus, $\overline{a p r}_{n}^{\prime \prime}$ satisfies (U8). 
Properties (L2') and (U2') imply (L3), (L4), (U3), (U4), and the following weaker version of (L5) and (U5):

$$
\begin{aligned}
& \underline{a p r}_{n}^{\prime}(X) \subseteq \bigcap_{x \notin X} \underline{a p r}_{n}^{\prime}(\sim\{x\}), \\
& \overline{a p r}_{n}^{\prime}(X) \supseteq \bigcup_{x \in X} \overline{a p r}_{n}^{\prime}(\{x\}) .
\end{aligned}
$$

Properties (L8) and (U8) imply (LU6), (L7), (U7), and (L8') and (U8'). Properties (L2) and (U2) imply (L3)-(L5), (U3)-(U5), and (L2') and (U2'). By Theorems 4 and 5 , the corresponding approximation operators satisfy such implied properties.

For an arbitrary neighborhood operator, the family of neighborhoods $\{n(x) \mid$ $x \in U\}$ may not be a covering of the universe. It is a covering of $U$ if and only if $n$ is an inverse serial neighborhood operator. Our formulation is therefore more general than the existing definitions using the notion of coverings. According to Table 1 , for an arbitrary binary relation $R$, none of the four families,

$$
\begin{aligned}
& C_{R_{p}}=\left\{R_{p}(x) \mid x \in U\right\}, \\
& C_{R_{s}}=\left\{R_{s}(x) \mid x \in U\right\}, \\
& C_{R_{p \wedge s}}=\left\{R_{p \wedge s}(x) \mid x \in U\right\}, \\
& C_{R_{p \vee s}}=\left\{R_{p \vee s}(x) \mid x \in U\right\},
\end{aligned}
$$

necessarily forms a covering of $U$. The families $C_{R_{s}}$ and $C_{R_{p \vee s}}$ are coverings if $R$ is an inverse serial. If the relation $R$ is serial, $C_{R_{p}}$ and $C_{R_{p \vee s}}$ are coverings. If $R$ is reflexive, all four families are coverings. Theorem 5 provides additional properties satisfied by approximation operators constructed from a covering.

With respect to a covering of the universe, Zakowski [25] used $\operatorname{apr}_{n}^{\prime}$ and $\overline{a p r}_{n}^{\prime \prime}$ as a pair of approximation operators. The same notion was also adopted by Wybraniec-Skardowska [22]. Orlowska [9-11] used the pair of of approximation operators $\underline{a p r}_{R_{p \vee s}}^{\prime}$ and $\overline{a p r}_{R_{p \vee s}}^{\prime \prime}$. A problem with such definitions is that they may not produce dual operators $[3,16]$.

\section{CONNECTIONS OF APPROXIMATION OPERATORS}

With respect to a neighborhood operator $n$, we have defined three pairs of dual approximation operators by using (I), (II'), and (II'). For comparison, we restate them using a similar format as follows: 
Table 2

An example of approximation operators

\begin{tabular}{|c||c|c||c|c||c|c|}
\hline$X$ & $\underline{a p r}_{n}$ & $\overline{a p r}_{n}$ & $\underline{a p r}_{n}^{\prime}$ & $\overline{a p r}_{n}^{\prime}$ & ${\underline{a p r_{n}^{\prime \prime}}}_{n} \overline{a p r}_{n}^{\prime \prime}$ \\
\hline \hline$\emptyset$ & $\emptyset$ & $\emptyset$ & $\emptyset$ & $\emptyset$ & $\emptyset$ & $\emptyset$ \\
$\{a\}$ & $\emptyset$ & $\{a\}$ & $\emptyset$ & $\{a\}$ & $\emptyset$ & $\{a, b\}$ \\
$\{b\}$ & $\{c\}$ & $\{a, c\}$ & $\{b\}$ & $\{a, b\}$ & $\emptyset$ & $\{a, b\}$ \\
$\{c\}$ & $\{b\}$ & $\{b\}$ & $\{c\}$ & $\{c\}$ & $\{c\}$ & $\{c\}$ \\
$\{a, b\}$ & $\{a, c\}$ & $\{a, c\}$ & $\{a, b\}$ & $\{a, b\}$ & $\{a, b\}$ & $\{a, b\}$ \\
$\{a, c\}$ & $\{b\}$ & $\{a, b\}$ & $\{c\}$ & $\{a, c\}$ & $\{c\}$ & $U$ \\
$\{b, c\}$ & $\{b, c\}$ & $U$ & $\{b, c\}$ & $U$ & $\{c\}$ & $U$ \\
$U$ & $U$ & $U$ & $U$ & $U$ & $U$ & $U$ \\
\hline
\end{tabular}

(I) $\quad \underline{a p r}_{n}(X)=\{x \in U \mid n(x) \subseteq X\}$, $\overline{\operatorname{apr}}_{n}(X)=\{x \in U \mid n(x) \cap X \neq \emptyset\}$;

$\left(\mathrm{II}^{\prime}\right) \quad \operatorname{apr}_{n}^{\prime}(X)=\{x \in U \mid \exists y[x \in n(y), n(y) \subseteq X]\}$, $\overline{a p r}_{n}^{\prime}(X)=\{x \in U \mid \forall y[x \in n(y) \Longrightarrow n(y) \cap X \neq \emptyset]\}$

$\left(\mathrm{II}^{\prime \prime}\right) \quad \underline{\operatorname{apr}}_{n}^{\prime \prime}(X)=\{x \in U \mid \forall y[x \in n(y) \Longrightarrow n(y) \subseteq X]\}$, $\overline{\operatorname{apr}}_{n}^{\prime \prime}(X)=\{x \in U \mid \exists y[x \in n(y), n(y) \cap X \neq \emptyset]\}$.

Definition (I) only uses the neighborhood of $x$ to decide if $x$ belongs to the lower or the upper approximation. In making the same decision, both definitions ( $\left.\mathrm{II}^{\prime}\right)$ and $\left(\mathrm{II}^{\prime \prime}\right)$ use a family of neighborhoods $\{n(y) \mid y \in U, x \in n(y)\}$. In some sense, they may be regarded as converse definitions. While definition ( $\left.\mathrm{II}^{\prime}\right)$ uses the existential quantifier for lower approximation and the universal quantifier for upper approximation, definition ( $\left.\mathrm{II}^{\prime \prime}\right)$ uses the opposite quantifiers. These three definitions produce distinct but related approximation operators.

Example 6 Consider a universe $U=\{a, b, c\}$. Suppose a neighborhood operator $n$ is given by:

$$
n(a)=\{a, b\}, \quad n(b)=\{c\}, \quad n(c)=\{b\} .
$$

It is a serial and an inverse serial neighborhood operator. Using definitions (I), $\left(\mathrm{II}^{\prime}\right)$, and $\left(\mathrm{II}^{\prime \prime}\right)$, we have three pairs of approximation operators in Table 2. 
From the example, one may observe that three pairs of approximation operators are different, although they are defined based on the same neighborhood operator. Operators $\underline{a p r}_{n}^{\prime}$ and $\overline{a p r}_{n}^{\prime}$ give tighter approximations than that of $\underline{a p r}_{n}^{\prime \prime}$ and $\overline{a p r}_{n}^{\prime \prime}$. For other operators, there is not such a relationship. If additional properties are imposed on neighborhood operators, much stronger relationships may be established.

Theorem 7 Suppose $n: U \longrightarrow 2^{U}$ is a neighborhood operator. If $n$ is inverse serial, the following relationships hold:

$$
\underline{a p r}_{n}^{\prime \prime}(X) \subseteq \underline{a p r}_{n}^{\prime}(X) \subseteq X \subseteq \overline{a p r}_{n}^{\prime}(X) \subseteq \overline{a p r}_{n}^{\prime \prime}(X)
$$

If $n$ is a reflexive, we have:

$$
\begin{gathered}
\underline{a p r}_{n}^{\prime \prime}(X) \subseteq \underline{a p r}_{n}(X) \subseteq \underline{a p r}_{n}^{\prime}(X) \subseteq X \subseteq \\
\overline{a p r}_{n}^{\prime}(X) \subseteq \overline{a p r}_{n}(X) \subseteq \overline{a p r}_{n}^{\prime \prime}(X)
\end{gathered}
$$

PROOF. (R1): For an inverse serial neighborhood operator $n$, by Theorems 4 and 5 we know that the set $X$ lies between its lower and upper approximations as defined by $\left(\mathrm{II}^{\prime}\right)$ and $\left(\mathrm{II}^{\prime \prime}\right)$. We only need to show $\underline{a p r}_{n}^{\prime \prime}(X) \subseteq \underline{a p r}_{n}^{\prime}(X)$. The other relation $\overline{a p r}_{n}^{\prime}(X) \subseteq \overline{a p r}_{n}^{\prime \prime}(X)$ can be obtained by duality. Assume $x \in \operatorname{apr}_{n}^{\prime \prime}(X)$. Since $n$ is inverse serial, there must exist a $y \in U$ such that $x \in \overline{n(y)}^{n}$. By assumption $x \in \underline{a p r}_{n}^{\prime \prime}(X)$ and definition of $\underline{a p r}_{n}^{\prime \prime}$, we can conclude that $n(y) \subseteq X$. It follows that $x \in \underline{a p r}_{n}^{\prime}(X)$. Therefore, $\underline{\underline{a p r}}_{n}^{\prime \prime}(X) \subseteq \underline{a p r}_{n}^{\prime}(X)$.

(R2) can be similarly proved.

Theorem 8 [16, page 660, Corollary of Theorem 3] Two pairs of lower and upper approximation operators defined by $\left(\mathrm{II}^{\prime}\right)$ and $\left(\mathrm{II}^{\prime \prime}\right)$ are equivalent if and only if the family $\{n(x) \neq \emptyset \mid x \in U\}$ forms a partition of the universe.

Theorem 9 Two pairs of lower and upper approximation operators defined by $(\mathrm{I})$ and $\left(\mathrm{II}^{\prime}\right)$ are equivalent if and only if the neighborhood operator $n$ is reflexive and transitive.

PROOF. $(\Longleftarrow) \quad$ By the reflexivity of $n$, we have $\underline{\operatorname{apr}}_{n}(X) \subseteq \underline{a p r}_{n}^{\prime}(X)$. Now we need to prove the reverse. Assume $x \in \underline{a p r}_{n}^{\prime}(X)$. There must exist a $y \in U$ such that $x \in n(y)$ and $n(y) \subseteq X$. By the transitivity of $n$, we have $n(x) \subseteq$ $n(y) \subseteq X$, which implies $x \in \underline{a p r}_{n}(X)$. Therefore, $\underline{a p r}{ }_{n}(X)=\underline{a p r}_{n}^{\prime}(X)$.

$(\Longrightarrow)$ Suppose $\frac{a p r}{U} n(X)=\underline{a p r}_{n}^{\prime}(X)$ and $\overline{a p r}_{n}(X)=\overline{a p r}_{n}^{\prime}(X)$ for all $X \subseteq$ $U$. For any $x \in \bar{U}$, by definition we have $x \in \overline{a p r}_{n}^{\prime}(\{x\})$. By assumption, 
$x \in \overline{a p r}_{n}(\{x\})$. It follows $x \in n(x)$, that is, $n$ is reflexive. For three elements $x, y, z \in U$, assume $y \in n(x)$ and $z \in n(y)$. By definition, $y \in \underline{a p r}_{n}^{\prime}(n(x))$. Thus, $y \in \operatorname{apr}_{n}(n(x))$. This implies $n(y) \subseteq n(x)$. Combining with $z \in n(y)$, we have $z \in n(x)$. Therefore, $n$ is transitive.

Theorem 10 Two pairs of lower and upper approximation operators defined by $(\mathrm{I})$ and $\left(\mathrm{II}^{\prime \prime}\right)$ are equivalent if and only if the neighborhood operator $n$ is symmetric and transitive.

PROOF. $(\Longleftarrow) \quad$ Assume $x \in \underline{a p r}_{n}^{\prime \prime}(X)$. Consider the family $O(x)=\{y \in$ $U \mid x \in n(y)\}$. If $O(x)=\emptyset$, by the symmetry of $n$ we can conclude $n(x)=\emptyset$, hence $x \in \underline{\operatorname{apr}}_{n}(X)$. If $O(x) \neq \emptyset$, by definition of $\underline{a p r}_{n}^{\prime \prime}$ we have $x \in n(y)$ and $n(y) \subseteq X$ for all $y \in O(x)$. By the transitivity of $n$, we have $n(x) \subseteq n(y) \subseteq X$. Thus, $x \in \operatorname{apr}_{n}(X)$. By summarizing the results for both cases, we obtain $\underline{a p r}_{n}^{\prime \prime}(X) \subseteq \underline{\operatorname{apr}}_{n}(X)$. Assume $x \in \underline{\operatorname{apr}}_{n}(X)$. We have $n(x) \subseteq X$. Consider any $y \in U$ with $x \in n(y)$. By the symmetry of $n$, it follows $y \in n(x)$. By the transitivity of $n$, we can conclude that $n(y) \subseteq n(x) \subseteq X$. According to definition, $x \in \underline{a p r}_{n}^{\prime \prime}(X)$. We thus proved $\underline{\operatorname{apr}}_{n}(X) \subseteq \underline{a p r}_{n}^{\prime \prime}(X)$. Therefore, $\underline{\operatorname{apr}}_{n}(X)=\underline{\operatorname{apr}}_{n}^{\prime \prime}(X)$.

$(\Longrightarrow)$ Suppose $\underline{a p r}_{n}(X)=\underline{a p r}_{n}^{\prime \prime}(X)$ and $\overline{a p r}_{n}(X)=\overline{a p r}_{n}^{\prime \prime}(X)$ for all $X \subseteq U$. For a pair $x, y \in \bar{U}$, assume $\overline{x \in} n(y)$. By Theorem 4, $\underline{a p r}_{n}^{\prime \prime}$ and $\overline{a p r}_{n}^{\prime \prime}$ satisfy (L9). It follows that $y \in \underline{a p r}_{n}^{\prime \prime}\left(\overline{a p r}_{n}^{\prime \prime}(\{y\})\right)$. Thus, $y \in \underline{a p r}_{n}\left(\overline{a p r}_{n}(\{y\})\right)$. From definition, we have $n(y) \subseteq \overline{\complement \overline{a p r}}_{n}(\{y\})$. By assumption, $\bar{x}_{\in}^{n} n(y) \subseteq \overline{a p r}_{n}(\{y\})$. It implies $y \in n(x)$, namely, $n$ is symmetric. For three elements $x, y, z \in$ $U$, assume $y \in n(x), z \in n(y)$. By definition, $x \in \underline{\operatorname{apr}}_{n}(n(x))$. Hence $x \in$ $\underline{a p r}_{n}^{\prime \prime}(n(x))$. By definition, for all $w, x \in n(w)$ implies $n(w) \subseteq n(x)$. From the symmetry of $n$ and assumption $y \in n(x)$, we have $x \in n(y)$, and hence $n(y) \subseteq n(x)$. Combining this with $z \in n(y)$, we obtain $z \in n(x)$. Therefore, $n$ must be transitive.

Based on the results of the last three theorems, Figure 1 summarizes the conditions under which two pairs of approximation operators are equivalent. With the condition:

(P) $\quad\{n(x) \neq \emptyset \mid x \in U\}$ is a partition,

we have:

$[$ reflexivity, $\mathrm{P}] \Longrightarrow$ symmetry,

[symmetry, transitivity, $\mathrm{P}] \Longrightarrow$ reflexivity,

[reflexivity, symmetry, transitivity] $\Longrightarrow \mathrm{P}$. 


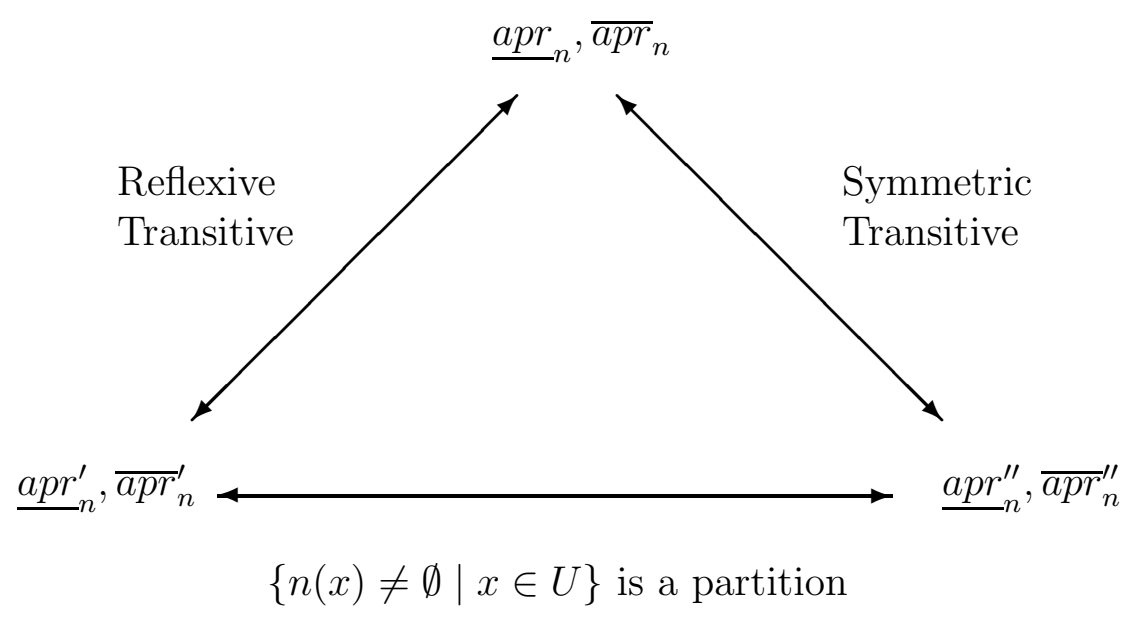

Fig. 1. Relationships between approximation operators

It should be pointed out that none of reflexivity, symmetry, and transitivity is necessary for $(\mathrm{P})$. For example, consider a universe $U=\{a, b, c\}$ with a neighborhood operator $n$ given by:

$$
n(a)=\{b\}, \quad n(b)=\{c\}, \quad n(c)=\{a\} .
$$

Obviously, $n$ is not reflexive, nor symmetric and transitive. Nevertheless, the family $\{\{b\},\{c\},\{a\}\}$ is a partition of $U$. A set of necessary and sufficient condition for $(\mathrm{P})$ is:

(P1) $n$ is inverse serial,

(P2) for all $x, y \in U$, either $n(x)=n(y)$ or $n(x) \cap n(y)=\emptyset$.

Condition (P1) is equivalent to saying that $\bigcup_{x \in U} n(x)=U$. The following Corollary follows from the last three theorems.

Corollary 11 Three pairs of lower and upper approximation operators defined by $(\mathrm{I}),\left(\mathrm{II}^{\prime}\right)$, and $\left(\mathrm{II}^{\prime \prime}\right)$ are equivalent if and only if $n$ is reflexive, symmetric, and transitive.

Neighborhood operators may be interpreted by using binary relations. Their properties are determined by the properties of binary relations. The results developed in this section may be alternatively stated using binary relations. 


\section{CONCLUSION}

We propose a binary relation based framework for the study of neighborhood systems and rough set approximations. Within this framework, these two notions may be formulated, interpreted, and compared. The class of 1neighborhood systems, in which each element has one neighborhood, are studied in detail. A binary relation is used to define and interpret such neighborhood systems. In particular, 16 distinct neighborhood operators are obtained from a binary relation. Properties of neighborhood operators are related to the properties of binary relations. Three generalizations of Pawlak approximation operators are suggested. Each of them captures different aspects in approximating a subset of the universe. One generalization is related to the notion of modal operators in modal logic. For this generalization, only the neighborhood of $x$ is used to decide the memberships of $x$ in the lower and upper approximation of a subset $X$. For the other two generalizations, the family of neighborhoods, $\{n(y) \mid x \in n(y)\}$, is used to decide the memberships of $x$. Conditions on neighborhood operators are identified, under which some or all of these approximation operators are equivalent.

The notions of neighborhood systems and approximation operators are useful in modeling approximation. In this paper, we only analyzed 1-neighborhood systems. A careful study of general neighborhood systems may produce a more powerful and useful tool in approximations.

\section{ACKNOWLEDGEMENTS}

The author is grateful for discussion with and suggestions from T.Y. Lin on neighborhood systems.

\section{References}

[1] B.F. Chellas, Modal Logic: An Introduction, Cambridge University Press, Cambridge, 1980.

[2] P.M. Cohn, Universal Algebra, Harper and Row Publishers, New York, 1965.

[3] L.T. Koczy, Review of "On a concept of rough sets" by Zakowski, Mathematical Review, 84i:03092 (1984).

[4] T.Y. Lin, Topological and fuzzy rough sets, in: R. Slowinski (Ed.), Intelligent Decision Support: Handbook of Applications and Advances of the Rough Sets Theory, Kluwer Academic Publishers, Boston, 1992, pp. 287-304. 
[5] T.Y. Lin, Neighborhood systems - application to qualitative fuzzy and rough sets, in: P.P. Wang (Ed.), Advances in Machine Intelligence 83 SoftComputing, Department of Electrical Engineering, Duke University, Durham, North Carolina, USA, 1997, pp. 132-155.

[6] T.Y. Lin and Q. Liu, Rough approximate operators: axiomatic rough set theory, in: W.P. Ziarko (Ed.), Rough Sets, Fuzzy Sets and Knowledge Discovery, Springer-Verlag, London, 1994, pp. 256-260.

[7] T.Y. Lin, Q. Liu, K.J. Huang, and W. Chen, Rough sets, neighborhood systems and approximation, in: Z.W. Ras, M. Zemankova, and M.L. Emrichm (Eds.), Methodologies for Intelligent Systems, 5: Proceedings of the fifth International Symposium on Methodologies of Intelligent Systems, Knoxville, Tennessee, October 25-27, 1990, North-Holland, New York, pp. 130-141.

[8] T.Y. Lin and Y.Y. Yao, Mining soft rules using rough sets and neighborhoods, in: Proceedings of the Symposium on Modelling, Analysis and Simulation, Computational Engineering in Systems Applications (CESA'96), IMASCS Multiconference, Lille, France, July 9-12, 1996, pp. 1996.

[9] E. Orlowska, Semantics of nondeterministic possible worlds, Bulletin of the Polish Academy of Sciences: Mathematics, 33:453-458 (1985).

[10] E. Orlowska, Semantics analysis of inductive reasoning, Theoretical Computer Science, 43:81-89 (1986).

[11] E. Orlowska, Kripke semantics for knowledge representation logics, Studia Logica, 49:255-272 (1990).

[12] Z. Pawlak, Rough sets, International Journal of Computer and Information Science, 11:341-356 (1982).

[13] Z. Pawlak, Rough classification, International Journal of Man-Machine Studies, 20:469-483 (1984).

[14] Z. Pawlak, Rough sets: a new approach to vagueness, in: L.A. Zadeh and J. Kacprzyk (Eds.), Fuzzy Logic for the Management of Uncertainty, John Wiley \& Sons, New York, 1992, pp. 105-118.

[15] Z. Pawlak, Hard and soft sets, in: W.P. Ziarko (Ed.), Rough Sets, Fuzzy Sets and Knowledge Discovery, Springer-Verlag, London, 1994, pp. 130-135.

[16] J.A. Pomykala, Approximation operations in approximation space, Bulletin of the Polish Academy of Sciences, Mathematics, 35:653-662 (1987).

[17] J.A. Pomykala, On definability in the nondeterministic information system, Bulletin of the Polish Academy of Sciences, Mathematics, 36:193-210 (1988).

[18] W. Sierpenski and C. Krieger, General Topology, University of Toronto, Toronto, 1956. 
[19] R. Slowinski and D. Vanderpooten, Similarity relation as a basis for rough approximations, in: P.P. Wang (Ed.), Advances in Machine Intelligence $\& 3$ SoftComputing, Department of Electrical Engineering, Duke University, Durham, North Carolina, USA, 1997, pp. 17-33.

[20] A. Wasilewska, Conditional knowledge representation systems - model for an implementation, Bulletin of the Polish Academy of Sciences: Mathematics, 37:63-69 (1987).

[21] S.K.M. Wong and Y.Y. Yao, Roughness theory, in: Proceedings of the Sixth International Conference on Information Processing and Management of Uncertainty in Knowledge-Based Systems, Granada, Spain, July 1-5, 1996, pp. 877-883.

[22] U. Wybraniec-Skardowska, On a generalization of approximation space, Bulletin of the Polish Academy of Sciences: Mathematics, 37:51-61 (1989).

[23] Y.Y. Yao, Two views of the theory of rough sets in finite universes, International Journal of Approximate Reasoning, 15:291-317 (1996).

[24] Y.Y. Yao and T.Y. Lin, Generalization of rough sets using modal logic, Intelligent Automation and Soft Computing, an International Journal, 2:103120 (1996).

[25] W. Zakowski, Approximations in the space $(U, \Pi)$, Demonstratio Mathematica, XVI:761-769 (1983). 